\begin{tabular}{l} 
SCIENCE \& TECHNOLOGY \\
Journal homepage: http://www.pertanika.upm.edu.my/ \\
\hline PERTANIKA
\end{tabular}

\title{
Performance Evaluation of Heuristics and Meta-Heuristics Traffic Control Strategies Using the UTNSim Traffic Simulator
}

\author{
Ng Kok Mun ${ }^{1 *}$ and Mamun Ibne Reaz ${ }^{2}$ \\ ${ }^{2}$ School of Electrical Engineering, College of Engineering, Universiti Teknologi MARA, \\ 40450 Shah Alam, Selangor, Malaysia \\ ${ }^{2}$ Department of Electrical, Electronic and Systems Engineering, Faculty of Engineering and Built Environment, \\ Universiti Kebangsaan Malaysia, 43600 UKM Bangi, Selangor, Malaysia
}

\begin{abstract}
In the past few decades, intelligent traffic controllers have been developed to responsively cope with the increasing traffic demands and congestions in urban traffic networks. Various studies to compare and evaluate the performance of traffic controllers have been conducted to investigate its effect on traffic performances such as its ability to reduce delay time, stops, throughputs and queues within a traffic network. In this paper, the authors aim to present another comparative study on heuristics versus meta-heuristics traffic control methods. To our knowledge, such comparison has not been conducted and could provide insights into a purely heuristic controller compared to meta-heuristics. The study aims to answer the research question "Can heuristics traffic control strategies outperformed meta-heuristics in terms of performance and computational costs?" For this purpose, a heuristics model-based control strategy (MCS) which was previously developed by the authors is compared to genetic algorithms (GA) and evolution strategy (ES) respectively on a nine intersections symmetric network. These control strategies were implemented via simulations on a traffic simulator called UTNSim for three different types of traffic scenarios. Performance indices such as average delays, vehicle throughputs and the computational time of these controllers were evaluated. The results revealed that the heuristic MCS outperformed GA and ES with superior performance in average delays whereas vehicle throughputs were

ARTICLE INFO

Article history:

Received: 14 February 2021

Accepted: 10 May 2021

Published: 31 July 2021

in close agreement. The computation time of the MCS is also feasible for real-time application compared to GA and ES that has longer convergent time.
\end{abstract}

DOI: https://doi.org/10.47836/pjst.29.3.38

$\overline{\text { E-mail addresses: }}$

ngkokmun@uitm.edu.my (Ng Kok Mun)

mamun.reaz@gmail.com (Mamun Ibne Reaz)

* Corresponding author
Keywords: Evolution strategy, genetic algorithm, heuristics algorithm, meta-heuristics algorithm 


\section{INTRODUCTION}

Intelligent traffic control systems have been developed throughout the decades to improve traffic flow and ease congestions in urban traffic networks. These traffic-responsive systems devise the most effective signal timing plans to optimize traffic flow by adjusting the splits, cycle time and offset at individual intersections. Traffic control methods can be categorized into model-based and non-model based traffic responsive control, route guidance and driver information systems, computational intelligence and agent-based methods ( $\mathrm{Ng}$ et al., 2013).

Computational intelligence includes artificial intelligence and meta-heuristics methods. Meta-heuristics intelligence methods such as ant colony optimization, bee colony optimization, particle swarm optimization, genetic algorithms, evolutionary programming, just to name a few, have been implemented to devise optimal signal timing plans. These methods aim to solve nonlinear programming problems through heuristic search of near optimal solutions. Tan et al. (2017) proposed a decentralized genetic algorithm (GA) with adaptive fitness function for minimizing average network delay. Each signalized intersection in the traffic network that comprised of four signalized intersections has its own controller. The GA assists the controllers to optimize traffic signal at morning peak hours by finding optimum solution through a process of fitness evaluation, selection and reproduction. Similar optimal solution of single objective function such as delay time is also conducted by Cao \& Luo (2019). In another work, GA has been implemented to solve a multiobjective signal optimization problem in a nine intersections network to maximize system throughputs, minimize travelling delays, enhance traffic safety, and avoid spillovers (Li \& Sun, 2018). The application of GA on multi objective function was performed by Davydov et al. (2019) on a road map fragment described using a microscopic traffic simulator.

In the area of model-based traffic responsive control, traffic models play an important role to predict future traffics parameters in the urban traffic network. Subsequently, control methods such as dynamic programming (Dang \& Rudova, 2018), linear programming (Li et al., 2014; Zhang et al., 2015; Grandinetti et al., 2015), quadratic programming (Aboudolas et al., 2010; Le et al., 2015) and multivariable regulators (Diakaki et al., 2002) were implemented to optimize traffic performance. These produced optimal solutions by solving a set of mathematical functions. On the other hand, model-based traffic responsive control can be solved by purely heuristics algorithms. Lammer \& Helbing (2008) developed decentralized heuristic control algorithm that anticipates current and future queues at the intersection. Le et al. (2015) proposed similar heuristics method called max-pressure (back-pressure) algorithms to regulate queues optimally in urban network. Other heuristics algorithms such as gating and perimeter control (Hajiahmadi et al., 2015) and demand balance control (Zhou et al., 2016) were implemented on oversaturated network based on 
the network fundamental diagram (NFD). Recently, the authors proposed a heuristic modelbased control strategy (MCS) for urban networks. The MCS improved performance of average delays up to $24 \%$ when compared with existing fixed-time system and successfully regulates queue spillbacks ( $\mathrm{Ng}$ et al., 2019).

Performance evaluation needs to be conducted to assess the performance of traffic networks with or without any control scheme. Performance evaluation has been conducted on traffic streams without any control schemes such as ramps and highways (Basri et al., 2020; Saha et al., 2015) and networks with control schemes (Al-Kandari et al., 2013; Stevanovic et al., 2017). Based on the literature review in the next section, performance evaluation of heuristics traffic control strategy in relative comparison with meta-heuristics methods had not been investigated. Such comparison is needed and may provide insights on the performance and behavior of heuristics against meta-heuristics control algorithms. Hence, the research question at hand is "Can heuristics traffic control strategies outperformed meta-heuristics in terms of performance and computation costs?" In view of this; a comparative study on the heuristics model-based control strategy (MCS) by Ng et al. (2019) against meta-heuristics methods in Hajbabaie et al. (2011) is presented in this paper.

The MCS heuristics algorithm is compared respectively with genetic algorithm (GA) and evolution strategy (ES) (a type of evolution programming) by Hajbabaie et al. (2011) on a nine-intersection symmetric case study network. The performance of the MCS, GA and ES is evaluated based on average delays, network throughputs and computation time. This study is conducted via simulations using a traffic simulator namely UTNSim (Ng et al., 2018; Ng et al., 2019). The next section in this paper provides the literature review and the research gaps. The method section gives an overview of the MCS and meta-heuristics control algorithms respectively, and the experimental setup of the nine-intersection symmetric case study network. Subsequently simulation results of controller effects on traffic performances are presented and discussed.

\section{LITERATURE REVIEW}

Whenever a control scheme is introduced to an urban traffic system; proper evaluation needs to be conducted on these proposed control schemes to ascertain their effectiveness in improving traffic performances. For example, Al-Kandari et al. (2013) compared vehicle throughputs by four different control methods on a single four phase signalized intersection. Stevanovic et al. (2017) compared adaptive traffic control with existing time-of-day (TOD) signal timing plans based on average delays, total delay travel time and number of stops. The study shown that adaptive traffic control reduced average delays, travel time and number of stops in recurring and non-recurring traffic conditions.

Many research works have been conducted in optimizing traffic control performances using meta-heuristics methods. Performance evaluation on these methods was done in 
relative comparison with other control methods. For example, Gao et al. (2019) compares the performance of an improved artificial bee colony algorithm (IABC) with mixed integer linear programming (MILP) on a simple network that comprised of three traffic intersections. The IABC underperformed in terms of optimal solutions but performed better in computational time compared to MILP. Toivio et al. (2020) implemented GA to derive optimized membership functions for a fuzzy controller. The GA optimized fuzzy controller outperformed the default fuzzy controller and the Tasman-Zanker field controller in terms of average delays. Doostali et al. (2020) derived optimal vehicle flow using GA and directed graph. The proposed method was compared to adaptive control and fixed-time system to evaluate queue length and waiting time at approaches. A review presented by Shaikh et al. (2020) shows that GA had been evaluated in comparison with the classical Webster timing scheme, MAXBAND and fixed-time systems. Many other comparative studies on meta-heuristics controller performances have been conducted such as GA versus conventional fuzzy logic (Tan et al., 2019) and comparison of GA, Hill Climbing and Simulated Annealing in Cantarella et al. (2015).

Heuristics control methods were also compared relatively with other control methods to investigate their effects on the road networks. The MCS heuristics algorithm which was developed by the authors had been compared previously with fixed-time system ( $\mathrm{Ng}$ et al., 2019). The MCS improved performance of average delays up to $24 \%$ when compared with existing fixed-time system and successfully regulates queue spillbacks. The hierarchical demand balance controller in Zhou et al. (2016) was compared to centralized controller and fixed-time system. It was found that the centralized controller has the best performance in terms of total time spent and total delay time of vehicles in the network. Le et al. (2015) compared their proposed backpressure policy algorithm against greedy and proportional policy algorithms in terms of network throughputs and congestion. Lammer and Helbing (2008) compared the proposed self-organized traffic control against optimization strategy, cycle-based strategy and stabilization strategy.

To the best of our knowledge, based on the review, performance evaluation of heuristics traffic control strategy in relative comparison with meta-heuristics methods had not been investigated. Such comparison is needed and may provide insights on the performance and behavior of heuristics control algorithms, which is also used to address non-linear traffic behaviors. Although meta-heuristics methods may provide optimal solutions systematically using an objective function; it can be weighed down by computational costs. Hence, the research question at hand is "Can heuristics traffic control strategies outperformed metaheuristics in terms of performance and computation costs?" As such comparison has not been conducted previously; this may bring insights into the benefits that may be offered by heuristics in comparison with meta-heuristics algorithms. 


\section{MATERIALS AND METHODS}

\section{Model-based Control Strategy}

Figure 1 provides an overview of the closed loop behaviour of the model-based control strategy (MCS) introduced by Ng et al. (2019). The MCS is an extension to traffic simulator called UTNSim developed by Ng et al. $(2015 ; 2016)$. The UTNSim comprised of the traffic network diagram editor (TNDE) and a traffic simulator based on the LWR-IM (Ng et al., 2018). On the other hand, the components of the MCS are NET-PREDICT, NET-CONTROL and INT-CONTROL. These algorithms were developed to perform network wide traffic predictions and coordinates control action at each of the signalized intersection within an urban traffic network (UTN) (Ng et al., 2019).

The NET-PREDICT perform predictions based on the LWR-IM traffic model to produce estimations of platoon size, platoon arrival time and queues at each signalized intersection within the UTN by using measured traffic data or simulated inputs. The NET-CONTROL algorithm consolidates these estimations from the NET-PREDICT and calculates queue spillbacks at each link entering a signalized intersection. Based on these traffic estimates, it coordinates network wide control by calling the INT-CONTROL heuristics algorithm at each signalized intersection. The control action at each signalized intersection is regulated by the INT-CONTROL heuristics algorithm that updates new phase durations to optimize traffic flow. In addition, this algorithm also regulates queue spillbacks at the intersections.

Figure 2 shows the predicted parameters at a signalized intersection by the NETPREDICT. The incoming vehicle platoons from adjacent intersection is described as clrd $_{a}$ and $c l r d_{b}$ which depicts the clearing time needed for the platoons to pass through the

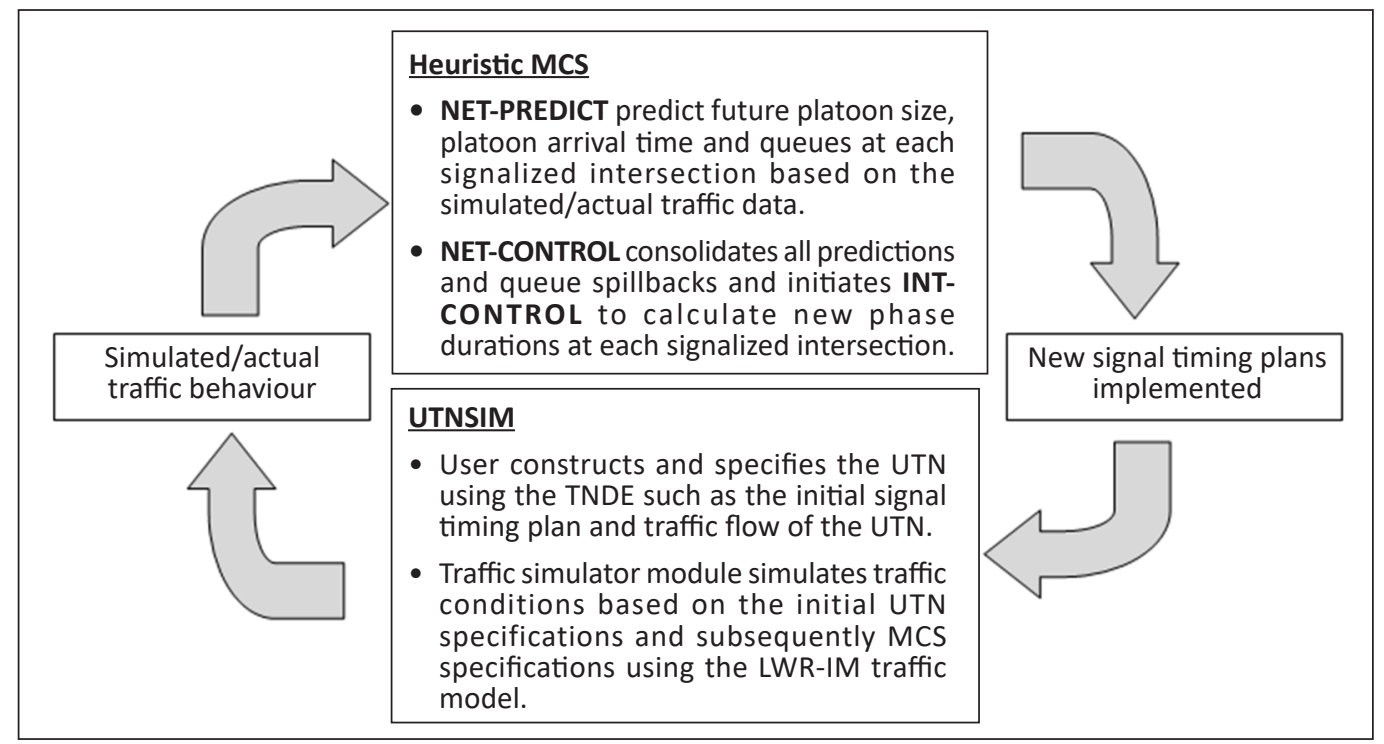

Figure 1. An overview of model-based control strategy in $\mathrm{Ng}$ et al. (2019) 


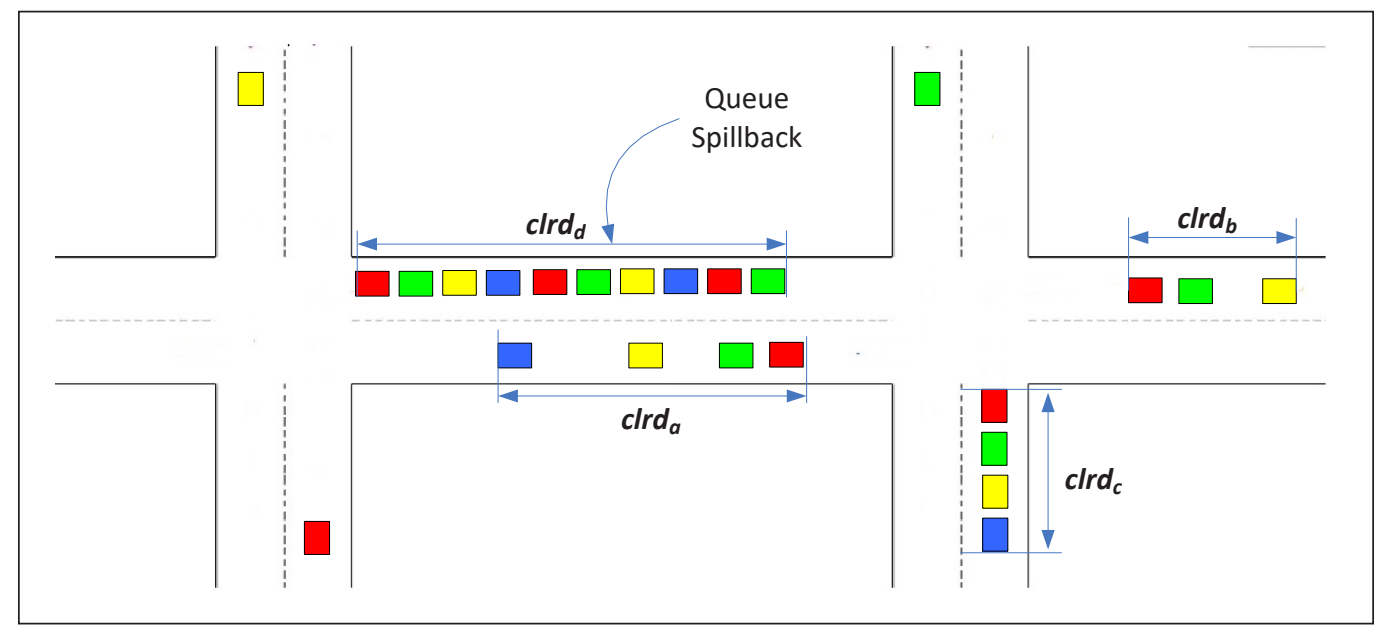

Figure 2. Predicted platoon and queue parameters at signalized intersections

intersection. Each platoon is described with estimated arrival time at the intersection. The length of the queue at the intersection is also described with a clearing time (i.e., $c l r d_{c}$ ). An intersection may also encounter queue spillbacks (Figure 2) when the queue occupies up to $80 \%$ of the link length with a predicted clearing time $c l r d_{d}$. The INT-CONTROL takes into consideration all these predicted parameters and adjust phase durations based on the proportionate method in responding to incoming vehicle platoons and queue clearing time. In addition, a queue regulation strategy is also embedded in the algorithm to regulate queue spillbacks. The detail description and functions of the INT-CONTROL heuristics algorithm can be referred in $\mathrm{Ng}$ et al. (2019).

\section{Genetic Algorithm (GA) and Evolution Strategy (ES)}

Hajbabaie et al. (2011) applied the GA and ES respectively to signal optimization problems in urban traffic networks. GA is a type of evolutionary computing inspired by evolutionary biology. The evolution process in GA includes inheritance, selection, crossover, and mutation. The GA created an initial population randomly or by mean of heuristics. Everyone in the population carries a fitness value calculated based on the objective function. Based on the fitness values, two parents are chosen from the initial population in the selection step. These parents' is crossed over, leading to two new individuals. The new individuals are mutated to form new individuals for the next generation. The fitness value of the newly created individuals will be evaluated. This whole process of selection, crossover, and mutation is repeated until certain termination criteria are met. On the other hand, the ES involves three steps i.e., recombination, mutation, and selection. In recombination, individuals among parents are selected and then recombined. In general, new individuals are generated from the recombinant via mutation and selection. 
Hajbabaie et al. (2011) applied the GA and ES respectively to the following signal optimization problem given by Equation 1:

$$
\operatorname{Max} \sum_{t=1}^{N} \sum_{i=1}^{N} \sum_{\varphi=2}^{\phi_{t i}} n_{i \varphi}^{t}-\sum_{t-1}^{N} \sum_{i=1}^{N} \sum_{\varphi=2}^{\phi_{t i}} \delta_{i \varphi}^{t} q_{i \varphi}^{t}
$$

subject to:

$$
\begin{array}{ll}
2 \leq \emptyset_{\mathrm{ti}} \leq 6 & t=0, \ldots ., T ; \quad i=1, \ldots \ldots, N \\
\operatorname{gmin}_{\mathrm{i} \varphi}^{\mathrm{t}} \leq \mathrm{g}_{\mathrm{i} \varphi}^{\mathrm{t}} \leq \operatorname{gmax}_{\mathrm{i} \varphi}^{\mathrm{t}} & t=0, \ldots ., T ; \quad i=1, \ldots \ldots, N ; \quad \varphi=1, \ldots \ldots, \emptyset \\
0 \leq \operatorname{off}_{\mathrm{i} \varphi}^{\mathrm{t}} \leq \mathrm{C}_{\mathrm{i}}^{\mathrm{T}} & t=0, \ldots \ldots, T ; \quad i=1, \ldots \ldots, N ; \quad \varphi=1, \ldots, \emptyset
\end{array}
$$

where:

$T$ is the number of study periods; $N$ is the total number of intersections; $\emptyset_{t i}$ is the number of signal phases at intersection $i$; at time period $t ; n_{i \varphi}^{t}$ is the total number of vehicles processed by intersection $i$, at time period $t$, in phase $\varphi ; q_{i \varphi}^{t}$ is the queue length at intersection $i$, at time period $t$, waiting to be served by phase $\varphi$; and $\delta_{i \varphi}^{t}$ is the penalty weight for queue length at intersection $i$, at time period $t$, waiting to be served by phase $\varphi$.

The chromosome or individuals in each respective GA or ES population, forms a vector which is a set of decisions variables. This vector contains signal timing parameters for each intersection $i$, for all defined time intervals $t$ such as phase plan $\varphi$, green time for each phase $\mathrm{g}_{\mathrm{i} \varphi}^{\mathrm{t}}$, and offset off $\mathrm{i} \varphi$. Hence, the implementation of the GA and ES respectively will produce decision variables that will optimize queue dissipation in the network based on Equation 1.

The generation of optimal signal timing plans according to Equation 1 from GA is illustrated in Figure 3(a) (Hajbabaie et al., 2011). The selection of parents was implemented using a tournament selection with a pressure of $6.7 \%$. There are various methods to perform crossover such as single-point, multi-point, two-point, and uniform crossover. In the crossover stage, Hajbabaie et al. (2011) implemented uniform crossover to select chromosome of the offspring probabilistically from one of the parents. To generate two individuals, two parents selected by the tournament selection were used. Next, bitwise mutation was applied by flipping each bit of the chromosome to produce new individuals according to the probability of mutation. These are repeated until the termination criterion.

In ES, a parent $\mu$ can be mutated to form a single individual notated as, $\mu+1$; or multiple individuals, $\mu+\lambda$ where $\lambda>1$. Hajbabaie et al. (2011) performed the $\mu+\lambda$ as illustrated in Figure 3(b). In the recombination stage, $\rho \geq 1$ individuals among parents are selected and then recombined. If $\rho>1$; recombinants are generated using both discrete and 
intermediate methods. However, if $\rho=1$ individual is selected; no recombination is done, and the new offspring is simply equal to its parent. In the mutation stage, the recombinants are mutated using single component mutation that results in concentric spheres around the parental state. The process of mutation will generate $\lambda$ descendants out of $\mu$ parents. The selection process will choose the $\mu$ best individuals out of the $\mu+\lambda$ individuals to form the next population. This process of recombination, mutation and selection is repeated until the termination criteria. The process terminated over a total of ten runs and the average fitness value for everyone is obtained. In each run, the solution must meet all the constraints, else it will be discarded, and a new solution is generated.

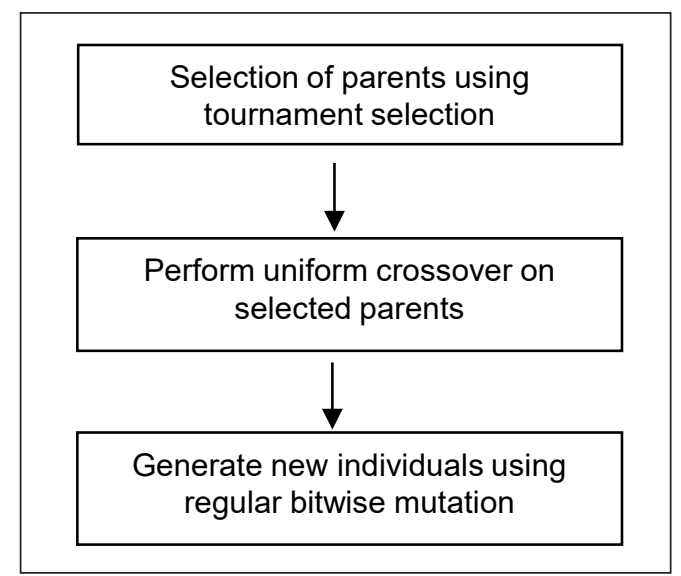

(a)

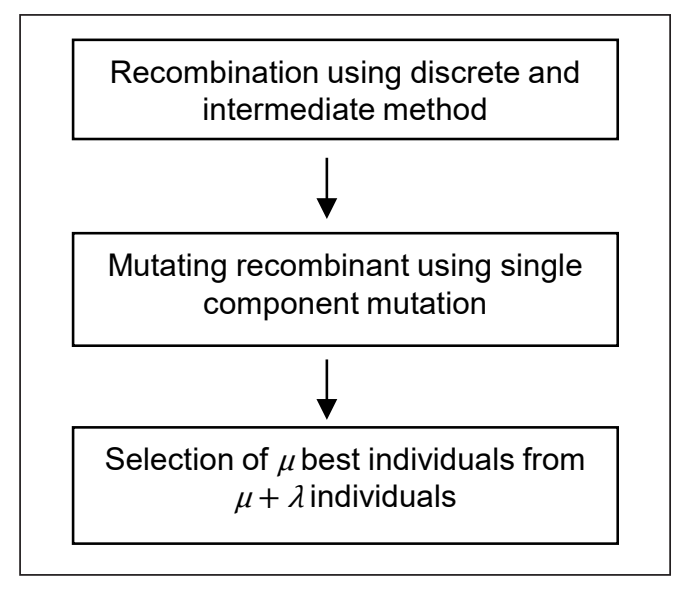

(b)

Figure 3. (a) Genetic Algorithm; and (b) Evolutionary System

\section{Experimental Setup}

The small hypothetical nine intersections symmetric case-study network in Hajbabaie et al. (2011) is employed in this work to evaluate the MCS and optimization compared to GA and ES algorithms. Figure 4 shows this small network which is symmetric in volume and geometry and composed of nine intersections (a three-by-three square) that is approximately $610 \mathrm{~m}$ (2000 ft) apart from each other. All streets are assumed to have two lanes (one per direction) and there are exclusive left-turn pockets, approximately $305 \mathrm{~m}(1000 \mathrm{ft})$ in length, at the intersections. The phase sequence in each intersection is shown in Figure 5. The turning rates at the intersections are $10 \%$ of vehicles turned right, $20 \%$ of the vehicles turned left and the rest went through. These turning percentages are estimated as the percentage of incoming volume from a single lane. A short study period of 15 minutes is conducted with the traffic demand fixed with the rate of $1000 \mathrm{veh} / \mathrm{h} / \mathrm{ln}$ at each entry point.

Based on the specifications in this traffic case-study, Hajbabaie et al. (2011) applied ES and GA (refer to GA and ES in previous section) to produce optimal signal settings for the network in Figure 4. The network in Figure 4 was described in VISSIM software. The GA 


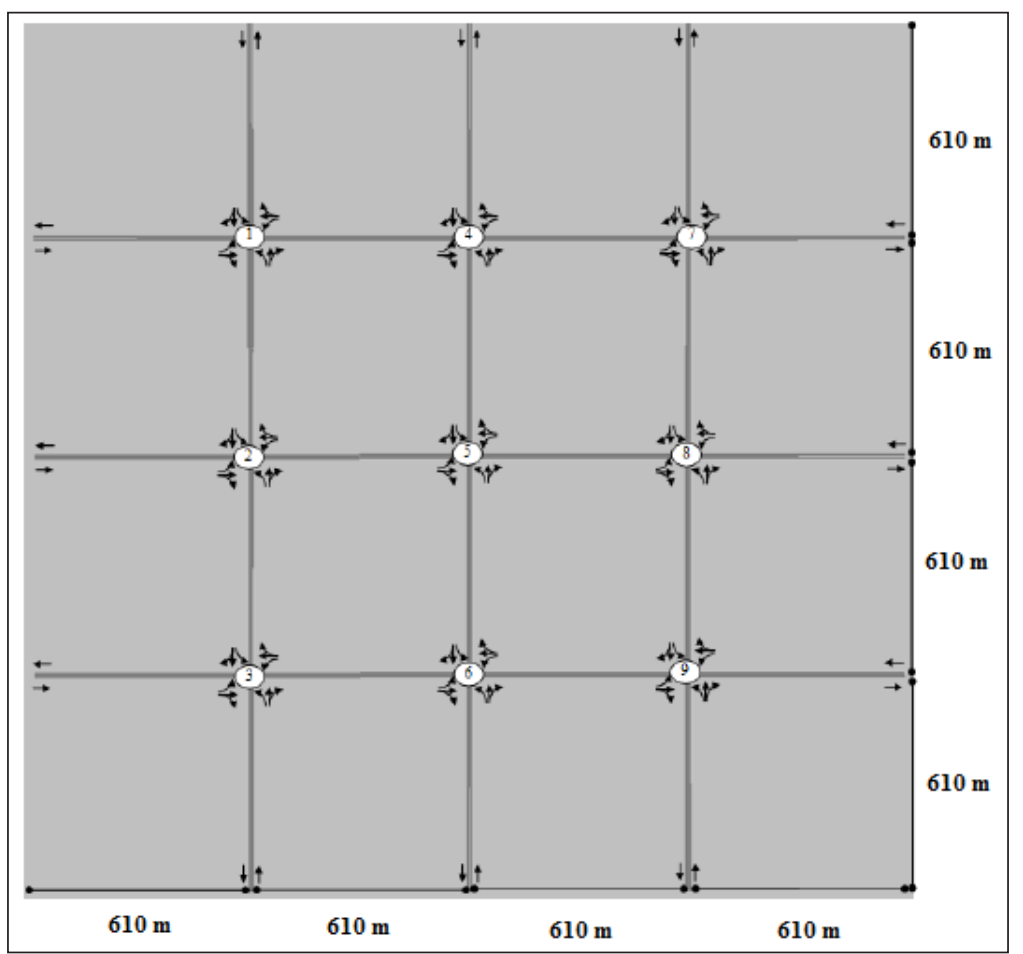

Figure 4. Nine intersections symmetric case study network as illustrated in Hajbabaie et al. (2011)

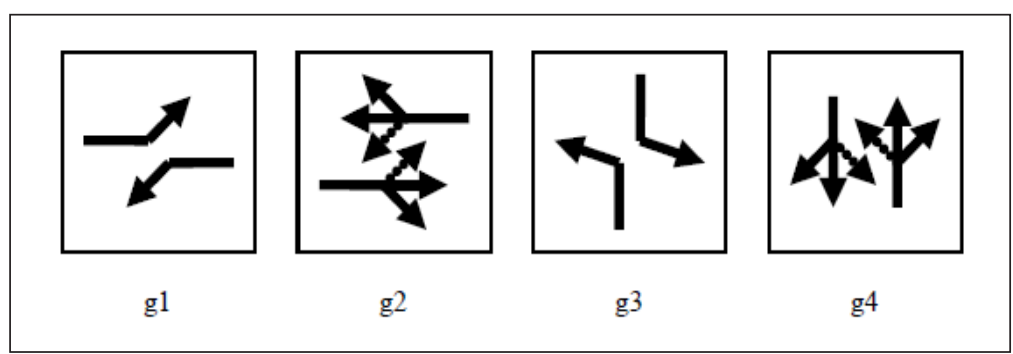

Figure 5. Phase sequence as illustrated in Hajbabaie et al. (2011)

and ES optimal timing plans were implemented in VISSIM. ES and GA were applied to the network in Figure 4 for three scenarios: (i) no overloading; (ii) 10\% network overloading and (iii) $20 \%$ network overloading. Simulations were performed for a 15 -minute analysis period in VISSIM based on the timing plans generated by ES and GA. The performances of the ES and GA approaches were analyzed and evaluated in terms of average delays, network throughputs, average number of stops, average speeds, efficient use of green time and queue overflows.

The comparative evaluation of the heuristic MCS with GA and ES comprise of the following steps: 
- The UTNSim simulator was used to specify the network in Figure 4 with similar specifications to simulate average delays and network throughputs by adopting similar timing plans produced by GA and ES for the three scenarios of overloading: (i) no overloading; (ii) 10\% network overloading and (iii) $20 \%$ network overloading. These GA and ES optimal timing plans can be referred in Hajbabaie et al. (2011). The average delays and network throughputs generated by UTNSim were compared with results from VISSIM to ascertain compatibility of both platforms. The simulated results in this step which have been conducted previously in Ng and Reaz (2017) had proven that UTNSim and VISSIM are compatible platforms.

- Upon ascertaining that UTNSim and VISSIM are compatible and comparable platforms for implementing the network; the relative comparison of traffic performances produced by the MCS with GA and ES approaches can be implemented using UTNSim.

- The heuristic MCS was implemented for no overload, $10 \%$ overload and $20 \%$ network overload, respectively. Simulations were conducted for an analysis period of 15 minutes. The optimized simulation was conducted with maximum green not exceeding $50 \%$ of the cycle time at each intersection; minimum green of $7 \mathrm{~s}$; the minimum cycle time set at $50 \mathrm{~s}$ whereas the maximum cycle time set at $70 \mathrm{~s}$. Average delays and network throughputs simulated for each situation using the MCS algorithm were compared with results produced by the GA and ES. The results were analyzed to observe if there is any improvement contributed by the heuristic MCS compared to these meta-heuristic approaches. Simulation using the UTNSim was conducted on a computer operating on an Intel Core i5 processor at $2.4 \mathrm{GHz}$.

- The third evaluation aims to tabulate and evaluate the average computation time (ACT) of the MCS in implementing each of the scenarios mentioned.

\section{RESULTS AND DISCUSSION}

The results simulated by the UTNSim using GA and ES timing plans are shown in Tables 1 and 2. Table 1 shows comparison of average delays between MCS and GA whereas Table 2 shows the comparison between MCS and ES.

Referring to Tables 1 and 2, further improvement on average delays produced by meta-heuristics methods is observed in all the scenarios simulated by the heuristic MCS. Figure 6 shows a graphical comparison of average delays produced by MCS, GA and ES respectively for all three scenarios. It is evident that the MCS has the best performance in terms of average delays. The MCS heuristic algorithm improved average delays for overloaded networks by more than $20 \%$, when compared with GA and ES. On the other 
Table 1

Comparison of MCS and GA average delays and network throughputs

\begin{tabular}{|c|c|c|c|c|c|c|c|}
\hline \multirow[b]{2}{*}{ Scenario } & \multicolumn{3}{|c|}{ Average delays } & \multicolumn{3}{|c|}{ Network Throughput } & \multirow{2}{*}{$\begin{array}{l}\mathrm{ACT} \\
(\mathrm{sec})\end{array}$} \\
\hline & $\begin{array}{c}\text { MCS } \\
\text { (s/veh) }\end{array}$ & $\begin{array}{c}\text { GA } \\
\text { (s/veh) }\end{array}$ & $\begin{array}{c}\text { Improvement } \\
(\%)\end{array}$ & $\begin{array}{c}\mathrm{MCS} \\
(\%)\end{array}$ & $\begin{array}{l}\text { GA } \\
(\%)\end{array}$ & $\begin{array}{r}\text { Diff. } \\
(\%)\end{array}$ & \\
\hline NOL & 177.48 & 194.74 & 8.86 & 71.55 & 70.47 & 1.08 & 4.04 \\
\hline $10 \% \mathrm{OL}$ & 200.36 & 250.87 & 20.13 & 75.68 & 74.80 & 0.88 & 4.54 \\
\hline $20 \% \mathrm{OL}$ & 208.34 & 263.33 & 20.88 & 79.89 & 79.11 & 0.78 & 4.35 \\
\hline
\end{tabular}

Table 2

Comparison of MCS and ES average delays and network throughputs

\begin{tabular}{|c|c|c|c|c|c|c|c|}
\hline \multirow[b]{2}{*}{ Scenario } & \multicolumn{3}{|c|}{ Average delays } & \multicolumn{3}{|c|}{ Network Throughput } & \multirow[b]{2}{*}{$\begin{array}{l}\mathrm{ACT} \\
(\mathrm{sec})\end{array}$} \\
\hline & $\begin{array}{l}\text { MCS } \\
\text { (s/veh) }\end{array}$ & $\begin{array}{c}\text { ES } \\
(\mathrm{s} / \text { veh })\end{array}$ & $\begin{array}{c}\text { Improvement } \\
(\%)\end{array}$ & $\begin{array}{c}\mathrm{MCS} \\
(\%)\end{array}$ & $\begin{array}{c}\mathrm{ES} \\
(\%)\end{array}$ & $\begin{array}{r}\text { Diff. } \\
(\%)\end{array}$ & \\
\hline NOL & 177.48 & 191.83 & 7.48 & 71.55 & 70.68 & 0.87 & 4.04 \\
\hline $10 \% \mathrm{OL}$ & 200.36 & 253.76 & 21.04 & 75.68 & 76.16 & -0.48 & 4.54 \\
\hline $20 \% \mathrm{OL}$ & 208.34 & 266.12 & 21.71 & 79.89 & 78.64 & 1.25 & 4.35 \\
\hline
\end{tabular}

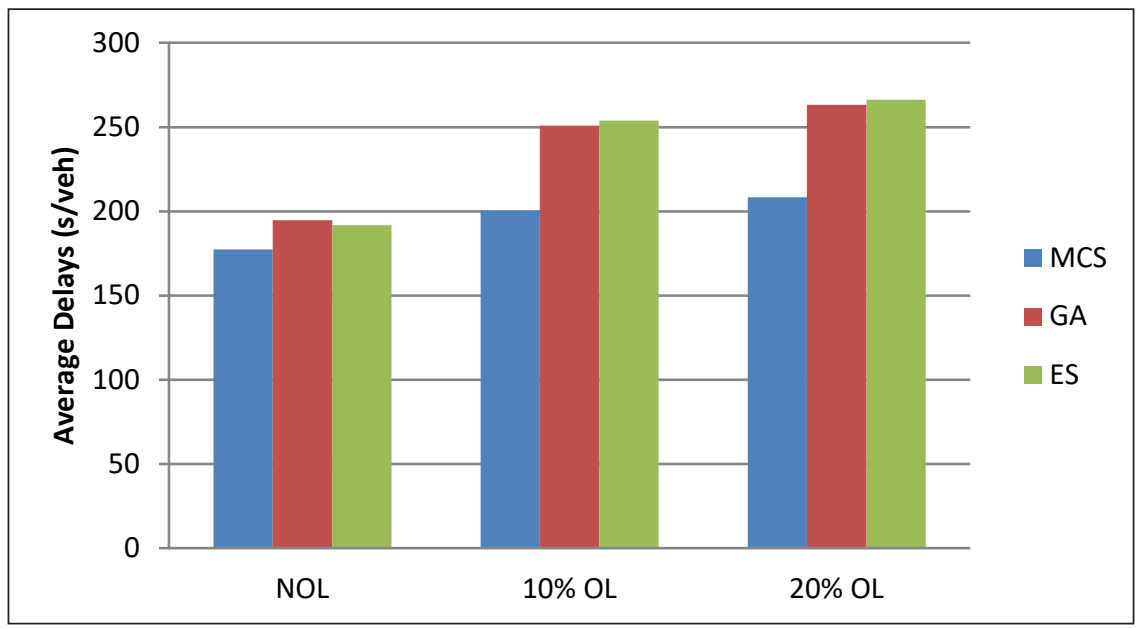

Figure 6. Average delays by MCS, GA and ES

hand, improvement of average delays for non-overloaded situations is $8.86 \%$ and $7.48 \%$ respectively.

The network throughput is defined as the total number of completed trips. This can be determined in terms of the total number of vehicles that had left the network at the end of the analysis period. For the network in this case study, 3000 vehicles entered the network at the end of the analysis period of 15 minutes. The network throughputs in Tables 1 and 2 show the percentage of vehicles out of the 3000 that completed their trips and left the network. It is observed that MCS, GA and ES network throughputs are similar and 


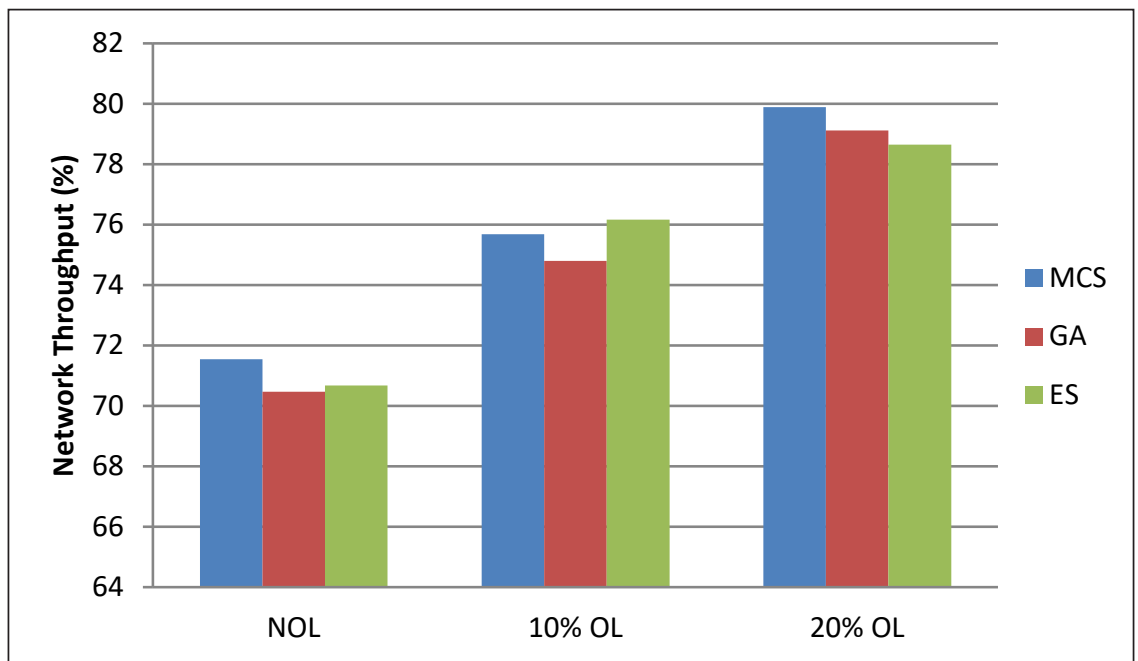

Figure 7. Vehicle throughputs by MCS, GA and ES

closer in agreement for all scenarios. Figure 7 shows a graphical comparison of network throughputs produced by MCS, GA and ES respectively for all three scenarios. Overall, MCS performed slightly better by producing higher throughputs compared to GA and ES in most scenarios except in $10 \%$ OL where it is $0.48 \%$ lesser compared to ES.

The improvement achieved by the MCS is primarily due to the consideration of both existing queues and incoming traffic at the respective intersection which are pre-processed into clusters by the NET-PREDICT algorithm. The NET-PREDICT utilizes schedule of an upstream intersection to predict the predicted output flows beyond the local prediction horizons of downstream neighbours. Due to the chain of propagation in the entire network, this provides a look-ahead in the optimization process that can avoid myopic mistakes at each local intersection. On the other hand, the GA and ES methods in Hajbabaie et al. (2011) only consider existing queues in its objective function. Myopic mistakes could be apprehended by not taking into consideration the anticipated incoming flows.

Based on the objective function and related constraints, the GA and ES searched for an optimal solution that converges to a single timing plan for the whole analysis period. The heuristic MCS breaks the entire analysis period into multiple cycles or time horizons. Based on predicted traffics from the NET-PREDICT for each time horizon, the algorithm generates an optimal timing plan for each time horizon. Hence, there are multiple sets of timing plans generated within the analysis period to cope with varying traffic demands. Table 3 shows generated timing plans for MCS, GA and ES respectively for $20 \%$ OL. For brevity, only signal timing plans for three intersections are shown. Each intersection comprised of four signal phases namely E-W L (East-West Left Turn); E-W R-T (EastWest Right-Through); N-S L (North-South Left Turn) and N-S R-T (North-South RightThrough), that indicates the direction of the vehicles going through the signal phase. The 
Table 3

MCS, GA and ES timing plans for 20\% OL

\begin{tabular}{|c|c|c|c|c|c|c|c|c|c|c|c|c|c|c|}
\hline \multirow{2}{*}{$\begin{array}{l}\text { Inter- } \\
\text { section }\end{array}$} & \multirow{2}{*}{$\begin{array}{l}\text { Signal } \\
\text { Phase }\end{array}$} & \multicolumn{11}{|c|}{ MCS Green Phase Duration at Cycle: } & \multirow{2}{*}{$\begin{array}{c}\text { GA } \\
\begin{array}{c}\text { All } \\
\text { cycles }\end{array}\end{array}$} & \multirow{2}{*}{$\begin{array}{c}\text { ES } \\
\begin{array}{c}\text { All } \\
\text { cycles }\end{array}\end{array}$} \\
\hline & & 1 & 2 & 3 & 4 & 5 & 6 & 7 & 8 & 9 & 10 & 11 & & \\
\hline \multirow{5}{*}{1} & E-W L & 11 & 7 & 8 & 22 & 15 & 29 & 25 & 7 & 25 & 22 & 7 & 6 & 6 \\
\hline & E-W R-T & 24 & 28 & 26 & 7 & 19 & 11 & 11 & 35 & 7 & 21 & 31 & 52 & 52 \\
\hline & N-S L & 11 & 7 & 8 & 21 & 14 & 23 & 26 & 7 & 13 & 13 & 7 & 6 & 6 \\
\hline & N-S R-T & 24 & 28 & 27 & 20 & 22 & 7 & 7 & 21 & 25 & 14 & 25 & 54 & 54 \\
\hline & Total CL & 70 & 70 & 69 & 70 & 70 & 70 & 69 & 70 & 70 & 70 & 70 & 118 & 118 \\
\hline \multirow{5}{*}{2} & E-W L & 7 & 7 & 22 & 18 & 26 & 21 & 11 & 11 & 7 & 11 & 16 & 6 & 6 \\
\hline & E-W R-T & 13 & 20 & 19 & 13 & 7 & 10 & 21 & 28 & 28 & 29 & 26 & 46 & 46 \\
\hline & N-S L & 21 & 18 & 8 & 22 & 26 & 26 & 10 & 7 & 7 & 7 & 7 & 6 & 6 \\
\hline & N-S R-T & 29 & 25 & 21 & 7 & 11 & 10 & 27 & 24 & 28 & 23 & 21 & 42 & 44 \\
\hline & Total CL & 70 & 70 & 70 & 60 & 70 & 67 & 69 & 70 & 70 & 70 & 70 & 100 & 102 \\
\hline \multirow{5}{*}{3} & E-W L & 11 & 8 & 7 & 21 & 13 & 23 & 12 & 35 & 35 & 31 & 20 & 6 & 6 \\
\hline & E-W R-T & 24 & 28 & 19 & 9 & 20 & 13 & 28 & 7 & 7 & 7 & 17 & 52 & 52 \\
\hline & N-S L & 11 & 7 & 15 & 19 & 9 & 21 & 7 & 7 & 9 & 11 & 8 & 6 & 6 \\
\hline & N-S R-T & 24 & 27 & 29 & 21 & 28 & 8 & 23 & 21 & 19 & 21 & 25 & 50 & 50 \\
\hline & Total CL & 70 & 70 & 70 & 70 & 70 & 65 & 70 & 70 & 70 & 70 & 70 & 114 & 114 \\
\hline
\end{tabular}

sum of all green phase durations equals the intersection total cycle length (Total CL). It can be observed that MCS green phase durations varies for each signal cycle according to traffic conditions whereas GA and ES green phases converged to a single value throughout the analysis period. Compared to a meta-heuristic search method, there is a stronger guarantee that the heuristics approach in the MCS that is tailored to flow information and adaptive timing plan could achieve better performance.

The ACTs in Tables 1 and 2 ranged between 4.04 to $4.35 \mathrm{~s}$ when the nine intersections network was simulated with an Intel Core i5 processor. Wey (2000) stated that the degree of forewarning in real-time networks is unlikely to exceed $15 \mathrm{~s}$. Hence, control actions that respond below $15 \mathrm{~s}$ can respond accurately in real-time situations. The ACTs achieved in this work has performed excellently beyond this 'benchmark'. In addition, the control time step for an MPC is normally below 5 minutes for traffic network control (Schutter et al., 2010); thus, the ACTs of the MCS is well suited for real-time application. In contrast, the GA and ES takes 4 minutes for each simulation run in VISSIM (Hajbabaie et al., 2011) which is inferior compared to MCS.

\section{CONCLUSION}

This comparative study on the performance of heuristics versus meta-heuristics control methods have shown that heuristics could outperformed meta-heuristics optimization 
control methods by producing greater reduction in average delays and improve traffic throughputs. This has shown somewhat that heuristics method could also provide optimal solutions in a nonlinear environment. In addition, due to extensive search in GA and ES for optimal solutions; the heuristics MCS fare better in computational time, which provide immediate benefits for its application in online real-time traffic control and optimization.

\section{ACKNOWLEDGEMENT}

This work is funded by the Ministry of Education Malaysia through the Fundamental Research Grant Scheme (FRGS) no: FRGS/1/ 2019/TK04/UITM/02/38.

\section{REFERENCES}

Aboudolas, K., Papageorgiou, M., Kouvelas, A., \& Kosmatopoulos, E. (2010). A rolling horizon quadraticprogramming approach to the signal control problem in large scale congested urban road networks. Transportation Research Part C: Emerging Technologies, 18(5), 680-694. https://doi.org/10.1016/j. trc.2009.06.003

Al-Kandari, A., Al-Shaikhli, I., \& Najaa, A. (2013). Comparative study between traffic control methods using simulation software. International Journal of Machine Learning and Computing, 3(5), 424-429. https:// doi.org/10.7763/ijmlc.2013.v3.353

Basri, N. S. H., Mohamed, N. A., Adnan, M. A., Mohamed, N. F., \& Zainuddin, N. H. (2020). Instantaneous speed ratio of traffic flowing through a merging area at kilometer 31.6 on the highway from Shah Alam to Kuala Lumpur. Pertanika Journal of Science \& Technology, 28(2), 565-578.

Cantarella, G. E., Luca, S. D., Pace, R. D., \& Memoli, S. (2015). Network signal setting design: Metaheuristic optimisation methods. Transportation Research Part C, 55, 24-45. https://doi.org/10.1016/j. trc.2015.03.032

Cao, H., \& Luo, J. (2019). Coordinated optimization control of regional traffic signals based on vehicle average delay model. In 2019 2nd International Conference of Intelligent Robotic and Control Engineering (IRCE) (pp. 72-77). IEEE Conference Publication. https://doi.org/10.1109/irce.2019.00022

Dang, Q. V., \& Rudová, H. (2018). Enhanced scheduling for real-time traffic control. In 2018 IEEE Symposium Series on Computational Intelligence (SSCI) (pp. 578-585). IEEE Conference Publication. https://doi. org/10.1109/ssci.2018.8628731

Davydov, I., Tolstykh, D., Kononova, P., \& Legkih, I. (2019). Genetic based approach for novosibirsk traffic light scheduling. In 2019 15th International Asian School-Seminar Optimization Problems of Complex Systems (OPCS) (pp. 31-36). IEEE Conference Publication. https://doi.org/10.1109/opcs.2019.8880158

Diakaki, C., Papageorgiou, M., \& Aboudolas, K. A. (2002). Multivariable regulator approach to trafficresponsive network-wide signal control. Control Engineering Practice, 10(2), 183-195. https://doi. org/10.1016/s0967-0661(01)00121-6

Doostali, S., Babamir, S. M., Dezfoli, M. S., \& Neysiani, B. S. (2020). IoT-based model in smart urban traffic control: Graph theory and genetic algorithm. In 2020 11th International Conference on Information 
and Knowledge Technology (IKT) (pp. 119-121). IEEE Conference Publication. https://doi.org/10.1109/ IKT51791.2020.9345623.

Gao, K., Wu, N., \& Wang, R. (2019). Meta-heuristic and MILP for solving urban traffic signal control. In 2019 International Conference on Industrial Engineering and Systems Management (IESM) (pp. 1-5). IEEE Conference Publication. https://doi.org/10.1109/iesm45758.2019.8948068.

Grandinetti, P., Garin, F., \& Canudas-de-Wit, C. (2015). Towards scalable optimal traffic control. In 2015 54th IEEE Conference on Decision and Control (CDC) (pp. 2175-2180). IEEE Conference Publication. https://doi.org/10.1109/cdc.2015.7402529

Hajbabaie, A., Medina, J. C., \& Benekohal, R. F. (2011). Traffic signal coordination and queue management in oversaturated intersections (No. 047IY02). NEXTRANS Center (US).

Hajiahmadi, M., Haddad, J., De Schutter, B., \& Geroliminis, N. (2015) Optimal hybrid perimeter and switching plans control for urban traffic networks. IEEE Transactions on Control Systems Technology, 23(2), 464478. https://doi.org/10.1109/tcst.2014.2330997

Lammer, S., \& Helbing, D. (2008). Self-control of traffic lights and vehicle flows in urban road networks. Journal of Statistical Mechanics: Theory and Experiment, P04019, 1-34. https://doi.org/10.1088/17425468/2008/04/p04019

Le, T., Kova'cs, P., \& Walton, N. (2015). Decentralized signal control for urban road networks. Transportation Research Part C: Emerging Technologies, 58, 431-450. https://doi.org/10.1016/j.trc.2014.11.009

Li, Y., Canepa, E., \& Claudel, C. (2014). Optimal traffic control in highway transportation networks using linear programming. In 2014 European Control Conference (ECC) (pp. 2880-2887). IEEE Conference Publication. https://doi.org/10.1109/ecc.2014.6862338

Li, X., \& Sun, J. (2018). Signal multiobjective optimization for urban traffic network. IEEE Transactions on Intelligent Transportation Systems, 19(11), 3529-3537. https://doi.org/10.1109/tits.2017.2787103

Ng, K. M. Reaz, M. B. I., \& Ali, M. A. M. (2019). Model-based control strategy for oversaturated traffic regimes based on the LWR-IM traffic model. IET Intelligent Transportation System, 13(5), 896-904. https://doi. org/10.1049/iet-its.2018.5381

Ng, K. M., Reaz, M. B. I., Ali, M. A. M., \& Chang, T. G. (2013). A brief survey on advances of control and intelligent systems methods for traffic-responsive control of urban networks. Tehnički vjesnik, 20(3), $555-562$.

Ng, K. M., \& Reaz, M. B. I. (2015). An integrated approach for platoon-based simulation and its feasibility assessment. PLoS ONE, 10(3), 1-25. https://doi.org/10.1371/journal.pone.0114406

Ng, K. M., \& Reaz, M. B. I. (2016). Platoon interactions and real-world traffic simulation and validation based on the LWR-IM. PLoS ONE, 11(1), 1-17. https://doi.org/10.1371/journal.pone.0144798

Ng, K. M., \& Reaz, M. I. (2017). Development and validation of the LWR-IM traffic simulator. In 2017 th IEEE International Conference on Control System, Computing and Engineering (ICCSCE) (pp. 40-44). IEEE Conference Publication. https://doi.org/10.1109/iccsce.2017.8284376

Ng, K. M., Reaz, M. B. I., \& Rahiman, M. H. F. (2018). UTNSim: A new traffic simulator based on the LWRIM mesoscopic traffic model. Journal of Engineering Science and Technology, 13(3), 589-608. 
Saha, P., Sarkar, A. K., \& Pal, M. (2015). Evaluation of performance measures of two-lane highways under heterogeneous traffic. Pertanika Journal of Science \& Technology, 23(2), 223-239.

Schutter, B. D., Hellendoorn, H., Hegyi, A., Van den Berg, M., \& Zegeye, S. K. (2010). Model-based control of intelligent traffic networks. Intelligent Systems, Control and Automation: Science and Engineering, 40(1), 277-310.

Shaikh, P. W., El-Abd, M., Khanafer, M., \& Gao, K. (2020). A review on swarm intelligence and evolutionary algorithms for solving the traffic signal control problem. In IEEE Transactions on Intelligent Transportation Systems (pp. 1-16). IEEE Conference Publication. https://doi.org/10.1109/TITS.2020.3014296.

Stevanovic, A., Dakic, I., \& Zlatkovic, M. (2017). Comparison of adaptive traffic control benefits for recurring and non-recurring traffic conditions. IET Intelligent Transport Systems, 11(3), 142-151. https://doi. org/10.1049/iet-its.2016.0032

Tan, M. K., Chuo, H. S. E., Yeo, K. B., Chin, R. K. Y., Huang, S., \& Teo, K. T. K. (2019). Decentralized traffic signal control for grid traffic network using genetic algorithm. In 2019 IEEE 6th International Conference on Engineering Technologies and Applied Sciences (ICETAS) (pp. 1-6). IEEE Conference Publication. https://doi.org/10.1109/icetas48360.2019.9117490

Tan, M. K., Chuo, H. S. E., Chin, R. K. Y., Yeo, K. B., \& Teo, K. T. K. (2017). Optimization of traffic network signal timing using decentralized genetic algorithm. In 2017 IEEE 2nd International Conference on Automatic Control and Intelligent Systems (I2CACIS) (pp. 62-67). IEEE Conference Publication. https:// doi.org/10.1109/i2cacis.2017.8239034

Toivio, T., Kosonen, I., \& Roncoli, C. (2020). A multilayer optimisation framework for policy-based traffic signal control. In 2020 Forum on Integrated and Sustainable Transportation Systems (FISTS) (pp. 347352). IEEE Conference Publication. https://doi.org/10.1109/FISTS46898.2020.9264870.

Wey, W. M. (2000). Model formulation and solution algorithm of traffic signal control in an urban network. Computers, Environment and Urban Systems, 24, 355-377.

Zhang, Y., Su, R., \& Gao, K. (2015). Urban road traffic light real-time scheduling. In 2015 54th IEEE conference on decision and control (CDC) (pp. 2810-2815). IEEE Conference Publication. https://doi.org/10.1109/ cdc.2015.7402642

Zhou, Z., Lin, S., \& Xi, Y. (2016). A hierarchical urban network control with integration of demand balance and traffic signal coordination. IFAC-PapersOnLine, 49(3), 31-36. https://doi.org/10.1061/41177(415)122 